\title{
Correction
}

\section{Correction: Ma et al., Kalirin-7 Is Required for Synaptic Structure and Function}

In the article "Kalirin-7 Is Required for Synaptic Structure and Function” by Xin-Ming Ma, Drew D. Kiraly, Eric D. Gaier, Yanping Wang, Eun-Ji Kim, Eric S. Levine, Betty A. Eipper, and Richard E. Mains, which appeared on pages 12368-12382 of the November 19, 2008 issue, the authors regret that the same magnified image of wild-type hippocampus appeared in Panel C (wild-type) and Panel F (improperly labeled knock-out) of Supplemental Figure 2. The correct image of knock-out hippocampus has been inserted in Panel F of the revised figure and is listed below.

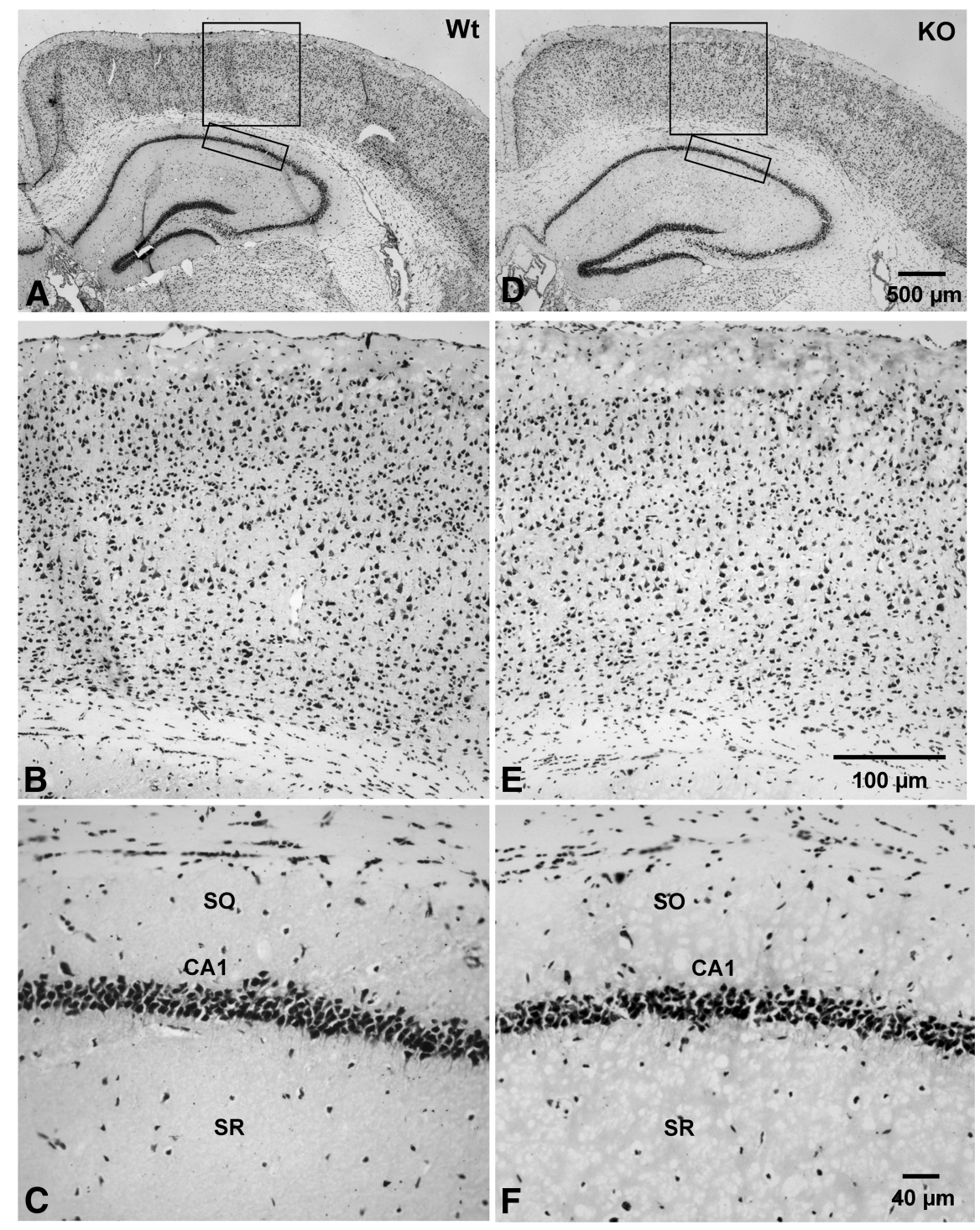

Supplemental Figure 2 . 